\title{
TITLE:
}

\section{An identification method of play model with input-dependent shape function}

\author{
$\operatorname{AUTHOR}(\mathrm{S}):$ \\ Matsuo, T; Shimasaki, M
}

\section{CITATION:}

Matsuo, T...[et al]. An identification method of play model with input-dependent shape function. IEEE TRANSACTIONS ON MAGNETICS 2005, 41(10): 3112-3114

\section{ISSUE DATE:}

2005-10

URL:

http://hdl.handle.net/2433/40004

\section{RIGHT:}

(c)2005 IEEE. Personal use of this material is permitted. However, permission to reprint/republish this material for advertising or promotional purposes or for creating new collective works for resale or redistribution to servers or lists, or to reuse any copyrighted component of this work in other works must be obtained from the IEEE. 


\title{
An Identification Method of Play Model With Input-Dependent Shape Function
}

\author{
Tetsuji Matsuo, Member, IEEE, and Masaaki Shimasaki, Member, IEEE \\ Graduate School of Engineering, Kyoto University, Kyoto 615-8510, Japan
}

\begin{abstract}
A product form of the input-dependent shape function is applied to the play model. This paper presents a method to determine the input-dependent shape function, thereby improving the representational accuracy of the play model. A simple data correction before identification is also introduced for the play model to avoid unnatural BH loops.
\end{abstract}

Index Terms-Hysteresis, identification, play model, silicon steel sheet, weighting function.

\section{INTRODUCTION}

$\mathbf{T}$ HE play model and the stop model [1], [2] are efficient hysteresis models that can be implemented more simply than the Preisach model [3], [4]. The play model with an input-independent shape function has been proven equivalent to the static scalar Preisach model [2], [5]. A previous study [6] has shown that the play and stop models with input-dependent shape functions are both equivalent to the nonlinear Preisach model proposed by Mayergoyz [3]. Mayergoyz proposed an identification method for the nonlinear Preisach model. This identification method uses second-order reversal curves of the hysteretic property. However, it is difficult to measure all second-order reversal curves that are required for identification.

On the other hand, a product form of the input-dependent shape function was proposed in [7] for the stop model. This form allows the stop model with input-dependent shape function to be identified using a two-step procedure: 1) determination of a weighting function; and 2) identification of the stop model with input-independent shape function.

This paper applies the product form of an input-dependent shape function to the play model and proposes an identification method of the play model with input-dependent shape function. This method determines the weighting function using the leastsquares method.

\section{Play Model With InPut-Dependent Shape Function}

The play model usually obtains a hysteretic output of magnetic flux density $B$ from an input of magnetic field $H$. However, the play model can also provide an output $H$ from input $B$ in the same way as the Preisach model, using the inverse distribution function method [8].

A discretized form of the play model with input-dependent shape function describes a hysteretic function having input $B$ as

$$
\begin{gathered}
H=P(B)=\sum_{m=1}^{2 M} f_{m}\left(p_{\zeta m}(B), B\right) \\
p_{\zeta}(B)=\max \left(\min \left(p_{\zeta}^{0}, B+\zeta\right), B-\zeta\right)
\end{gathered}
$$

where $p_{\zeta}$ is the play hysteron operator having width $\zeta, 2 M$ is the number of hysterons, $\zeta_{m}=(m-1) B_{S} /(2 M), p_{\zeta}^{0}$ is the value

Digital Object Identifier 10.1109/TMAG.2005.854892 of $p_{\zeta}$ at the previous time point, $f_{m}$ is the input-dependent shape function for $p_{\zeta m}$, and $B_{\mathrm{S}}$ is the saturation magnetic flux density.

A product form of the input-dependent shape function

$$
f_{m}(p, B)=w(B) f_{m 0}(p)
$$

was proposed in [7] for the stop model, where $w(B)$ is called the weighting function. Using (3) for the play model (1), relation (4) is obtained as

$$
\frac{P(B)}{w(B)}=P_{0}(B)=\sum_{m=1}^{2 M} f_{m 0}\left(p_{\zeta m}(B)\right)
$$

where $P_{0}(B)$ represents the play model having an input-independent shape function $f_{m 0}$.

Identification of the play model using (3) requires determination of functions $w(B)$ and $f_{m 0}$. This paper proposes a method for determination of $w(B)$ using a least-squares method after a discussion of identification of $f_{m 0}$.

\section{IDENTIFICATION OF Play MODEL With INPUT-INDEPENDENT SHAPE FUNCTION}

\section{A. Identification Using the Everett Function}

Because of its equivalence to the Preisach model, a play model with input-independent shape function can be identified from the Everett function [4], [9] as follows.

The Everett function $E(\alpha, \beta)$ is defined from symmetric loops as

$$
E(\alpha, \beta)= \begin{cases}h_{\mathrm{S}}^{-}(\beta, \beta)-h_{\mathrm{S}}^{-}(\beta, \alpha) & (\alpha+\beta \geq 0) \\ h_{\mathrm{S}}^{+}(|\alpha|, \beta)-h_{\mathrm{S}}^{+}(|\alpha|, \alpha) & (\alpha+\beta \leq 0)\end{cases}
$$

where $h_{\mathrm{S}}^{+}(a, B)$ and $h_{\mathrm{S}}^{-}(a, B)$ are, respectively, the ascending and descending branches of the symmetric loop with amplitude $a$.

A piecewise linear shape function as (6) is used for identification

$$
\begin{array}{r}
f_{m 0}(p)=f_{m 0}\left(p_{m, j-1}\right)+\mu_{m, j}\left(p-p_{m, j-1}\right) / \Delta p \\
\quad\left(p_{m, j-1} \leq p \leq p_{m, j}, j=1, \ldots, 2 M-m+1\right) .
\end{array}
$$

Therein, $p_{m, j}=-B_{\mathrm{S}}+\zeta_{m}+j \Delta p, \Delta p=B_{\mathrm{S}} / M$, and $\mu_{m, j}=$ $f_{m 0}\left(p_{m, j}\right)-f_{m 0}\left(p_{m, j-1}\right)$. The Everett function gives $\mu_{m, j}$ as

$$
\begin{aligned}
\mu_{m, j}= & E\left(b_{j-1}, b_{k}\right)-E\left(b_{j-1}, b_{k-1}\right)-E\left(b_{j}, b_{k}\right) \\
& +E\left(b_{j}, b_{k-1}\right) \\
k= & m+j-1
\end{aligned}
$$



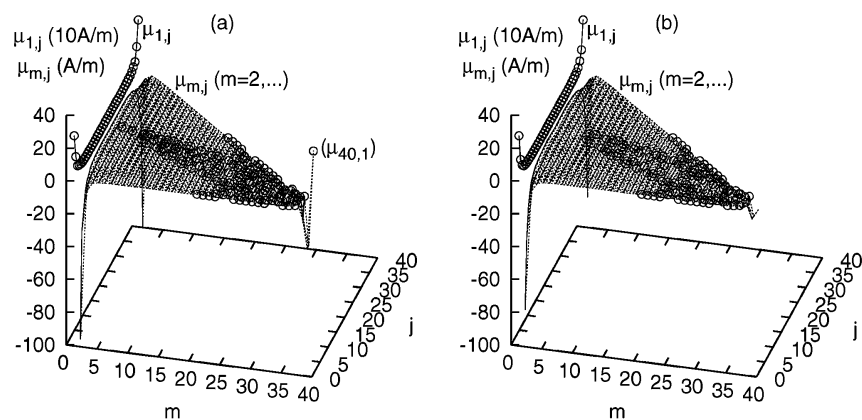

Fig. 1. Distribution of $\mu_{m, j}$. (a) From raw data. (b) From corrected data.
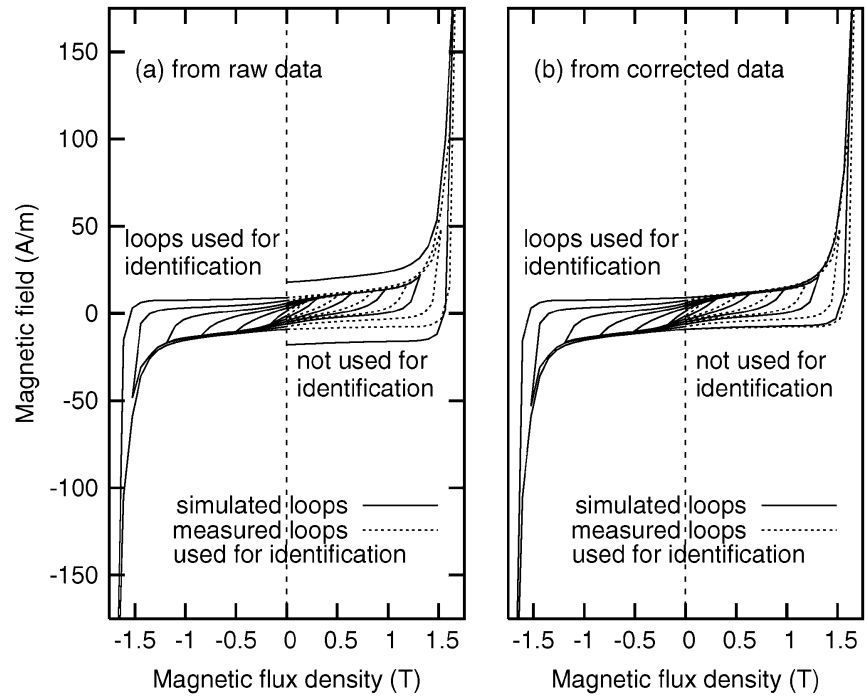

Fig. 2. Simulated symmetric BH loops.

where $b_{k}=k B_{\mathrm{S}} / M-B_{\mathrm{S}}(k=0, \ldots, 2 M)$ and $E\left(b_{j}, b_{j-1}\right)=$ 0 .

\section{B. An Improvement of Identification Method}

BH loops of a grain-oriented silicon steel sheet (JIS 30P105) along its rolling direction are represented by the play model. The play model is identified from 20 symmetric $\mathrm{BH}$ loops, of which amplitudes are given as

$$
a=c B_{\mathrm{S}} / M, \quad c=1, \ldots, M, \quad M=20 .
$$

Fig. 1(a) depicts the distribution of $\mu_{m, j}$, which corresponds to the distribution function of the Preisach model. Marks "O" indicate the points where $\mu_{m, j}$ is positive. Many of $\mu_{m, j}$ for irreversible component $(m \geq 2)$ are negative, giving a phase lead of output $H$ from input $B$.

Fig. 2(a) shows simulated BH loops of the silicon steel sheet. Several BH loops that were not used for identification $(c=3.5,7.5,11.5,15.5,19.5)$ are shown on the right half of Fig. 2(a), whereas loops used for identification $(c=2,6,10,14,18,20)$ are shown on the left. The play model accurately represents $\mathrm{BH}$ loops that were used for identification. However, one simulated loop that was not used for identification has a wider loop width than the major loop $(c=20)$. This failure is mainly caused by measured data that satisfy

$$
h_{S}^{+}\left(a_{m-1}, B_{m-1}\right)>h_{\mathrm{S}}^{+}\left(a_{m}, B_{m-1}\right)
$$

where $a_{n}=n \Delta B, B_{m}=m \Delta B$ and $\Delta B=B_{\mathrm{S}} / M$. This relation (10) yields a positive $\mu_{2 m, M-m+1}$ as

$$
\mu_{2 m, M-m+1}=2\left\{h_{\mathrm{S}}^{+}\left(a_{m-1}, B_{m-1}\right)-h_{\mathrm{S}}^{+}\left(a_{m}, B_{m-1}\right\}\right.
$$

where symmetric condition $h_{\mathrm{S}}^{+}(a, B)=-h_{\mathrm{S}}^{+}(a,-B)$ is assumed. Fig. 1(a) shows that $\mu_{40,1}$ has a large positive value.

To prevent $\mu_{2 m, M-m+1}$ from becoming positive, the values of $h_{\mathrm{S}}^{+}\left(a_{m-1}, B_{m-1}\right)$ and $h_{S}^{-}\left(a_{m-1},-B_{m-1}\right)$ are replaced by those of $h_{S}^{+}\left(a_{m}, B_{m-1}\right)$ and $h_{S}^{-}\left(a_{m},-B_{m-1}\right)$, respectively, before identification. Fig. 1(b) depicts the distribution of $\mu_{m, j}$ after this data correction, where the large positive value of $\mu_{m, j}$ disappears. Fig. 2(b) shows BH loops given by identification after data correction, where $\mathrm{BH}$ loops are naturally represented.

\section{Identification of a Play Model With INPUT-DEPENDENT SHAPE FUNCTION}

\section{A. Determination of Weighting Function}

For a hysteretic function $H(B)$ to be represented accurately by the play model using (3), $H(B) / w(B)$ should have the congruency property as the Preisach model requires [2]. This property is used to determine an optimal $w(B)$.

First-order reversal curves from negative saturation give the Everett function [4] $E(\alpha, \beta)$ as

$$
E(\alpha, \beta)=h_{\mathrm{R}}(\beta, \beta)-h_{R}(\beta, \alpha)
$$

where $h_{\mathrm{R}}(a, B)$ is the first-order reversal curve having maximum input $a$. The congruency property requires that the Everett function given by (12) coincides with that of (5).

Accordingly, an optimal weighting function is given by a least-squares method that determines $v_{n}(n=0, \ldots, M-1)$ to minimize the following:

$$
\begin{aligned}
\sum_{m=-M+1}^{-1} & \sum_{n=m+1}^{-m}\left[v_{|n|}\left\{h_{\mathrm{S}}^{+}\left(a_{|m|}, B_{n}\right)-h_{\mathrm{R}}\left(a_{n}, B_{n}\right)\right\}\right. \\
& \left.-v_{|m|}\left\{h_{\mathrm{S}}^{+}\left(a_{|m|}, B_{m}\right)-h_{\mathrm{R}}\left(a_{n}, B_{m}\right)\right\}\right]^{2} \\
& +\sum_{n=1}^{M-1} \sum_{m=-n+1}^{n-1}\left[v_{n}\left\{h_{\mathrm{S}}^{-}\left(a_{n}, B_{n}\right)-h_{\mathrm{R}}\left(a_{n}, B_{n}\right)\right\}\right. \\
& \left.-v_{|m|}\left\{h_{\mathrm{S}}^{-}\left(a_{n}, B_{m}\right)-h_{\mathrm{R}}\left(a_{n}, B_{m}\right)\right\}\right]^{2} \\
& +\left(\sum_{m=0}^{M-1} v_{m}-1\right)^{2} .
\end{aligned}
$$

The last term in (13) is introduced to yield a nonzero solution. A piecewise linear weighting function is given from $v_{n}$ by

$$
\begin{aligned}
w(B)= & w_{m}+\left(w_{m+1}-w_{m}\right)\left(B-B_{m}\right) / \Delta B \\
& \left(B_{m} \leq B \leq B_{m+1}\right) \\
w_{m}= & 1 / v_{|m|}(m=-M+1, \ldots, M-1) \\
w_{ \pm M}= & 2 w_{ \pm(M-1)}-w_{ \pm(M-2)} .
\end{aligned}
$$

\section{B. Numerical Examination}

Identification from symmetric $\mathrm{BH}$ loops gives a highly accurate representation of symmetric BH loops, as shown in Fig. 2(b). Accordingly, this subsection compares simulated and measured first-order reversal curves from negative saturation 

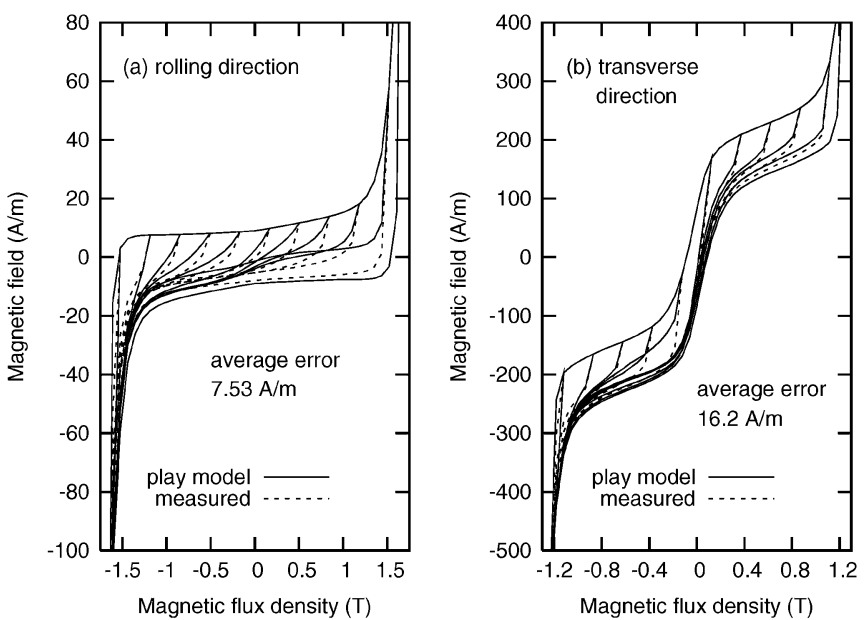

Fig. 3. Simulated first-order reversal curves and average representation errors given by the play model with weighting function 1 ).
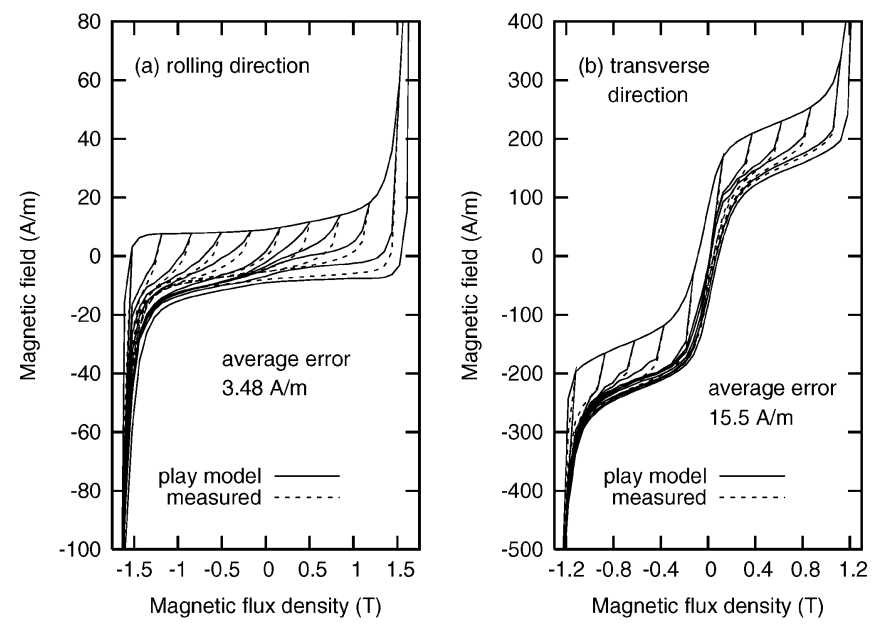

Fig. 4. Simulated first-order reversal curves and average representation errors given by the play model with weighting function 2).

of the steel sheet 30P105 along its rolling and transverse directions.

The following weighting functions are examined here:

1) $w(B)=1$;

2) $w(B)$ that minimizes $(13)$;

3) $w(B)=h_{\mathrm{S}}^{+}\left(B_{\mathrm{S}}, B\right)-h_{\mathrm{S}}^{-}\left(B_{\mathrm{S}}, B\right)$.

Function 1) gives an input-independent shape function. Function 3) uses the width of major BH loop [7], which was proposed for the stop model as an effective weighting function.

Figs. 3-5 respectively show simulated reversal curves that are given by the play model using the three weighting functions above. Figs. 3-5 also list the respective average discrepancy from the measured $H$. Discrepancies between measured and simulated $H$ are large in Fig. 3. Fig. 4 shows that the proposed function 2) improves the representation accuracy of the play model. Fig. 5 shows that function 3 ) is also effective, as in the case of the stop model. Comparison of Figs. 4(b) and 5(b) shows that function 2) does not always give the smallest representation error. Function 3 ) can be a reasonable choice because of its simplicity.
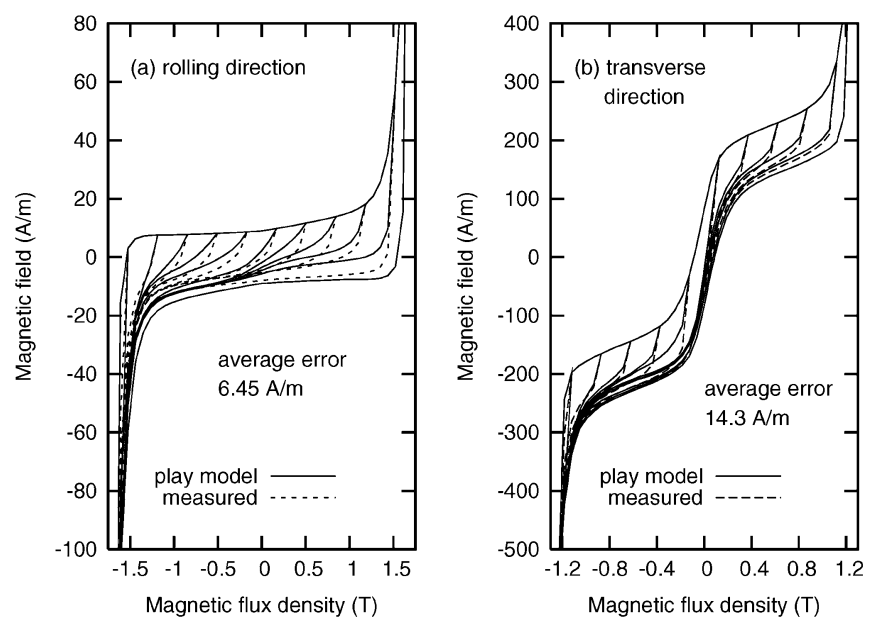

Fig. 5. Simulated first-order reversal curves and average representation errors given by the play model with weighting function 3 ).

\section{CONCLUSION}

- This paper introduced a simple data correction before identification of the play model; it prevents the play model from yielding unnatural BH loops.

- A method for determining the weighting function was proposed. The method improves the play model's representation accuracy.

- A simple weighting function using a major loop width is also effective, as in the case of the stop model.

\section{ACKNOWLEDGMENT}

This work was supported in part by the Japan Society for the Promotion of Science under Grant-in-Aid for Scientific Research (C) 16560243.

\section{REFERENCES}

[1] M. A. Krasnosel'skii and A. V. Pokrovskii, Systems with Hysteresis. Berlin, Heidelberg, Germany: Springer-Verlag, 1989.

[2] S. Bobbio, G. Miano, C. Serpico, and C. Visone, "Models of magnetic hysteresis based on play and stop hysterons," IEEE Trans. Magn., vol. 33, no. 11, pp. 4417-4426, Nov. 1997.

[3] I. D. Mayergoyz, Mathematical Models of Hysteresis. New York: Springer-Verlag, 1991.

[4] E. D. Torre, Magnetic Hysteresis. New York: IEEE Press, 1999.

[5] M. Brokate, "Some mathematical properties of the Preisach model for hysteresis," IEEE Trans. Magn., vol. 25, no. 7, pp. 2922-2924, Jul. 1989.

[6] T. Matsuo and M. Shimasaki, "Representation theorems for stop and play models with input-dependent shape functions," IEEE Trans. Magn., vol. 41, no. 5, pp. 1548-1551, May 2005.

[7] T. Matsuo, Y. Terada, and M. Shimasaki, "Stop model with input-dependent shape function and its identification methods," IEEE Trans. Magn., vol. 40, no. 7, pp. 1776-1783, Jul. 2004.

[8] N. Takahashi, S. Miyabara, and K. Fujiwara, "Problems in practical finite element analysis using Preisach hysteresis model," IEEE Trans. Magn., vol. 35, no. 3, pp. 1243-1246, Mar. 1999.

[9] T. Matsuo, D. Shimode, Y. Terada, and M. Shimasaki, "Application of stop and play models to the representation of magnetic characteristics of silicon steel sheet," IEEE Trans. Magn., vol. 39, no. 5, pp. 1361-1364, May 2003.

Manuscript received February 7, 2005. 